\title{
Soto syndrome: a rare overgrowth disorder
}

\author{
D C de Silva ${ }^{1}$, N de Leeuw ${ }^{2}$, R Gunasekera ${ }^{3}$
}

Ceylon Medical Journal 2013; 58: 40-42

\section{Introduction}

Childhood overgrowth is rare and may be associated with other abnormalities including facial dysmorphic features, congenital malformations, developmental delay, risk of malignancies and possible recurrence in another family member. Establishing an accurate diagnosis is essential to decide on long term follow up with regard to the risk of malignancies which vary according to the underlying diagnosis. This is a case report of a boy with an overgrowth syndrome called Soto syndrome (cerebral gigantism).

\section{Case report}

The patient is the only child of non consanguineous Sinhalese parents. He was born after an uneventful pregnancy and an induced vaginal delivery at term plus one week in a west Asian country. His birth weight (2.94 kg), head circumference $(33 \mathrm{~cm})$ and length $(50 \mathrm{~cm})$ were within normal limits. Medical records regarding his neonatal period are not available, but it appears that he was suspected of being asphyxiated. Brain imaging using MRI was reported as being consistent with this diagnosis while a CT scan performed in Sri Lanka showed dilated ventricles.

He was referred for a syndrome diagnosis at 15 months of age when mild motor and speech developmental delay were noted (standing with support only, no speech or babbling). His vision and hearing were normal. His weight $(12 \mathrm{~kg})$ and head circumference $(50 \mathrm{~cm})$ were between the 91st and 98th centiles while his height (83 $\mathrm{cm}$ ) was on the 2nd. He had mild frontal bossing, bitemporal hair loss, relative marocephaly and down slanting eyes (Figure 1). His bone age was advanced. His karyotype was normal. Echocardiography demonstrated normal valves and no septal anomalies. Renal ultrasound scanning indicated mild, bilateral renal pelvicalyceal fullness.

His clinical features were suggestive of Soto syndrome. Since the first description of this syndrome in a group of developmentally delayed children with overgrowth, clinical diagnostic criteria have been established [1,2]. These include the triad of typical dysmorphism (as noted in this child), significant overgrowth with head size and height being above +2SD from birth and variable degrees of developmental delay.

This boy fulfilled two of the criteria but a definite diagnosis was not possible due to his growth parameters being atypical.

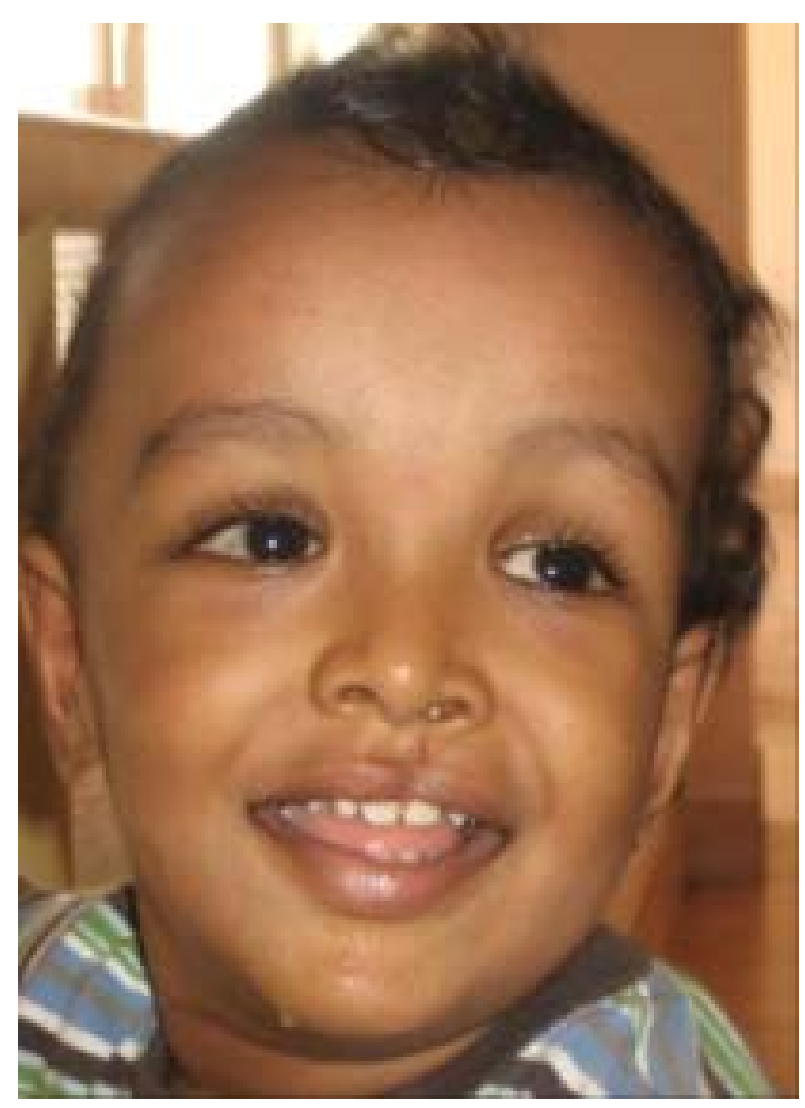

Figure 1. Patient at the age of 15 months. Note incomplete cleft lip, frontal bossing, bitemporal balding with high frontal hairline and down slanting eyes.

A mutation of the NSD1 gene (nuclear receptor SET domain -1 gene) is found in more than $90 \%$ of cases fulfilling the Soto syndrome diagnostic criteria [3]. Mutations of NSD1 were rare (12\%) among Japanese cases but in around half, a deletion of chromosome 5 q35 including the NSD1 gene has been identified [4]. Cases with a deletion displayed less obvious overgrowth [3].

${ }^{1}$ Department of Physiology, Faculty of Medicine, University of Kelaniya, Sri Lanka, ${ }^{2}$ Array Diagnostics, Centre for Genetics, Nijmegen, Netherlands, ${ }^{3}$ Plastic Surgery Unit, Lady Ridgway Hospital, Colombo, Sri Lanka.

Correspondence: DdeS, e-mail: <deepthidesilva@lycos.com>. Received 3 February and revised version accepted 9 August 2012. Competing interests: none declared. 


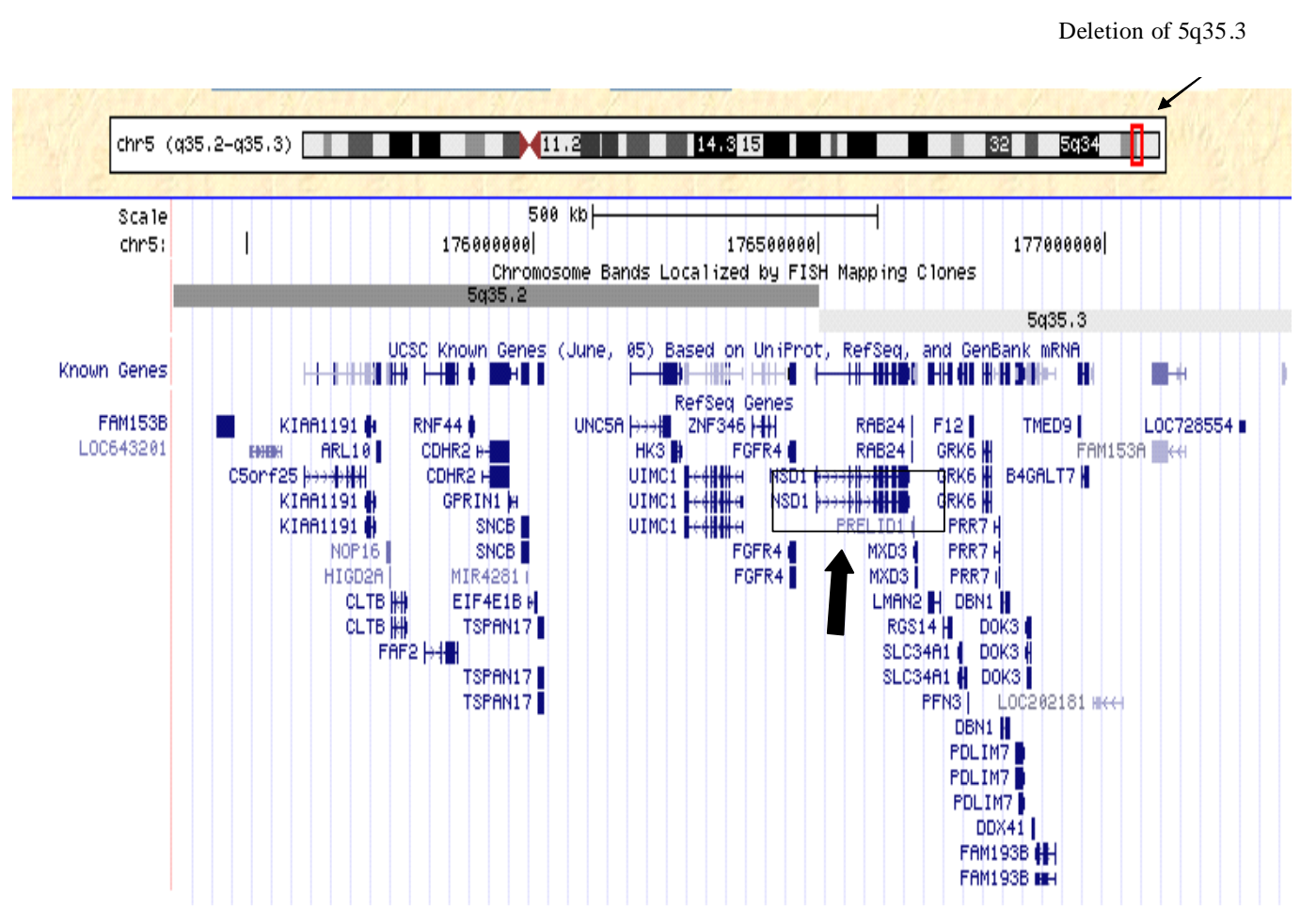

Figure 2. Ideogram of chromosome 5 indicating the deleted region at $5 q 35.3$ (top line) and the deleted genes within this region including NSD1 (solid arrow).

Based on the clinical findings and review of the molecular findings, it was decided to investigate this child for a $5 q 35$ deletion in preference to a mutation test. We elected to use microarrays as this technique is highly sensitive. Using an affymetrix SNP array with an average genome wide resolution of $200 \mathrm{~kb}$, a $1.64 \mathrm{Mb}$ deletion [(SNP_A-1935968->SNP_A2162864)x1] was identified. The deletion is flanked by a region of repeat sequences and is a common site for deletions in Soto syndrome. This patient's deletion is estimated to encompass 40 genes including the NSD1 gene (Figure 2).

\section{Discussion}

Syndrome diagnosis and confirmation using genetic testing is a complex process. Genetic testing is expensive and time consuming; adopting a rational approach to testing is therefore necessary. Genetic testing for Soto syndrome is especially useful in establishing the diagnosis in cases not fulfilling the clinical diagnostic criteria or in which features of another overgrowth syndrome may be present. In a few rare families, Soto syndrome can be inherited and testing will help to determine the risk of recurrence in another family member. In most cases fulfilling the clinical diagnostic criteria, genetic testing is not essential for diagnosis but would give more accurate information on recurrence risks for siblings or off spring of affected cases.
The clinical suspicion of Soto syndrome would usually be confirmed by NSD1 gene mutation testing but a literature review suggested that this child may have a chromosome 5q35 deletion which would not be apparent on mutation testing. The deletion that is commonly described in this condition is $1.9 \mathrm{Mb}$ and would not be identifiable using conventional karyotyping (typically detects deletions around 3-5 Mb). The submicroscopic deletion could have been confirmed using fluorescent in situ hybridization (FISH), multiple ligation probe amplification (MLPA) or microarrays.

Microarrays are currently the diagnostic method of choice in patients with developmental delay of unknown cause and multiple congenital malformations, but are expensive and unavailable locally [5,6]. Array diagnosis is less time consuming and more accurate in determining the size of the deletion than other methods and enables a whole genome assessment. Array detected anomalies require expertise from both the laboratory and the clinicians to determine their significance.

This child's clinical features were suggestive of Soto syndrome, a rare overgrowth disorder associated with variable degrees of developmental delay and characteristic dysmorphic features especially between the ages of 1 to 6 years. The facial features change with time and the growth rate normalizes but macrocephaly persists even in adults. Review of early childhood photographs is useful for 
clinicians suspecting the condition in an older child or adult.

The NSD1 gene is a transcription regulator implicated in regulating histone protein methylation, nuclear receptor binding and chromatin-chromatin interactions. The mechanism of how its insufficiency causes overgrowth is not known. A variety of different mutation mechanisms cause Soto syndrome but there is no correlation between the type or position of the mutation and the severity of the clinical features [7].

The deletion identified in this child is within a commonly affected region on chromosome $5 q 35$. There is a region of repetitive DNA that flanks the NSD1 gene region and the deletion associated with Soto syndrome is presumed to arise by intrachromosomal recombination of this region. In the Japanese population, there is a commonly identified inversion of this region that may predispose to the deletion in the offspring of carriers but its prevalence among other populations remains to be elucidated [8]. The deleted chromosome is usually the paternal copy suggesting that recombination in this region is commoner during male meiosis [3]. In general, deletion cases (with multiple genes deleted as in this case with around 40 deleted genes) are more likely to have developmental delay and less overgrowth [7].

Some overgrowth syndromes are associated with higher rates of embryonal and other tumours but this risk is low in Soto syndrome and regular cancer screening is not recommended [3]. Soto affected cases have a higher incidence of some congenital anomalies such as cardiac and renal malformations (not identified in this case) although interestingly, cleft lip which is present in him, is not a commonly recognised feature. Progressive scoliosis may be a future problem and this boy will need follow up for this [3].

Neither of his parents have clinical features of Soto syndrome, but they may have a balanced chromosome rearrangement that may predispose their next child to this condition. A balanced rearrangement (such as the inversion described earlier) will require karyotyping to identify it but, they have declined this test at present. This child's future offspring are at $50 \%$ risk of also having this condition.
This case reinforces the need for a careful clinical assessment in children with developmental delay and dysmorphic features as an accurate clinical diagnosis can guide investigations to diagnose the condition as well as guide further management. Genetic testing to confirm or refute the presumed clinical diagnosis is useful whenever possible and this is especially helpful in atypical cases.

\section{References}

1. Sotos JF, Dodge PR, Muirhead DD, et al. A syndrome of excessively rapid growth and acromegalic features and a nonprogressive neurologic disorder. New England Journal of Medicine 1964; 271: 109-16.

2. Cole TR, Hughes H. Sotos syndrome: a study of the diagnostic criteria and natural history. Journal of Medical Genetics 1994; 31: 20-32.

3. Tatton-Brown K, Rahman N. Soto syndrome. European Journal of Human Genetics 2007; 15: 264-71.

4. Kurotaki N, Harada N, Shimokawa O, et al. Fifty microdeletions among 112 cases of Sotos syndrome: Low copy repeats possibly mediate the common deletion. Human Mutation 2003; 22: 378-87.

5. Miller DT, Adam MP, Aradhya S, et al. Consensus statement: chromosomal microarray is a first-tier clinical diagnostic test for individuals with developmental disabilities or congenital anomalies. American Journal of Human Genetics 2010, 86: $749-64$.

6. Manning M, Hudgins L. Array-based technology and recommendations for utilization in medical genetics practice for detection of chromosomal abnormalities. Genetic Medicine 2010, 12: 742-5.

7. Tatton-Brown K, Douglas J, Coleman K, Baujat G, Cole T, Das S, et al. Genotype- phenotype associations in Soto syndrome: an analysis of 266 individuals with NSD1 aberrations. American Journal of Human Genetics 2005; 77: 193-204.

8. Visser R, Shimokawa O, Harada N, et al. Identification of a $3.0 \mathrm{~kb}$ major recombination hotspot in patients with Sotos syndrome who carry a common $1.9 \mathrm{Mb}$ microdeletion. American Journal of Human Genetics 2005; 76: 52-67. 